\title{
Evolution of Nuclear Shells due to the Tensor Force
}

\author{
Takaharu Otsuka, ${ }^{1,2,3, *}$ Toshio Suzuki, ${ }^{4}$ Rintaro Fujimoto, ${ }^{1}$ Hubert Grawe, ${ }^{5}$ and Yoshinori Akaishi ${ }^{6}$ \\ ${ }^{1}$ Department of Physics, University of Tokyo, Hongo, Bunkyo-ku, Tokyo 113-0033, Japan \\ ${ }^{2}$ Center for Nuclear Study, University of Tokyo, Hongo, Bunkyo-ku, Tokyo 113-0033, Japan \\ ${ }^{3}$ RIKEN, Hirosawa, Wako-shi, Saitama 351-0198, Japan \\ ${ }^{4}$ Department of Physics, Nihon University, Sakurajosui, Setagaya-ku, Tokyo 156-8550, Japan \\ ${ }^{5}$ GSI, D-64291, Darmstadt, Germany \\ ${ }^{6} \mathrm{KEK}$, Oho, Tsukuba-shi, Ibaraki 305-0801, Japan
}

(Received 22 February 2005; published 30 November 2005)

\begin{abstract}
The monopole effect of the tensor force is presented, exhibiting how spherical single-particle energies are shifted as protons or neutrons occupy certain orbits. An analytic relation for such shifts is shown, and their general features are explained intuitively. Single-particle levels are shown to change in a systematic and robust way, by using the $\pi+\rho$ meson exchange tensor potential, consistently with the chiral perturbation idea. Several examples are compared with experiments.
\end{abstract}

DOI: $10.1103 /$ PhysRevLett.95.232502

Shell structure characterizes finite quantum many-body systems. Atomic electrons confined by the Coulomb potential are subject to a well-known shell structure. For nuclei, since Mayer and Jensen [1], the shell structure has played a major role in clarifying nuclear structure. Recently, much progress has been made in the structure of exotic nuclei, which have rather extreme ratios of proton number $(Z)$ to neutron number $(N)$. Naturally, what new features can be found in their shell structure is of great and general interest.

Along these lines, in this Letter, we present the variation of the nuclear shell structure due to the tensor force. The nucleon-nucleon $(N N)$ interaction is originally due to meson exchange processes as predicted by Yukawa [2], and its tensor-force part is one of the most distinct manifestations of this meson exchange origin. As we shall show, the tensor force does indeed change the shell structure in a unique and robust way throughout the nuclear chart. The tensor force has been discussed over many decades. Its contribution to the spin-orbit splitting has been discussed, for instance, by Arima and Terasawa in terms of the second-order perturbation [3]. The importance of the tensor force for the nuclear binding energy has been demonstrated, for instance, by Pudliner et al. [4]. We shall show, in this Letter, how single-particle levels are changed systematically by the tensor force in the first order. The tensor force itself has certainly been included in various numerical calculations as one of the channels of the realistic nuclear force. Its first-order effect was discussed in individual cases, e.g., for ${ }^{15} \mathrm{C}$ and ${ }^{16} \mathrm{O}$ in [5]. In other early attempts, a possible tensor-force effect on the reduction of the spinorbit splitting was discussed in [6] with an example in the Os- $\mathrm{Pb}$ region [7]. The purpose of this Letter is, however, to present, for the first time, an analytic relation and a robust general feature, as well as concrete examples in close relation to experiments.

The change of the shell structure, or the shell evolution, may have different origins. We focus upon the shell evo-
PACS numbers: $21.60 .-\mathrm{n}, 21.10 .-\mathrm{k}, 21.30 . \mathrm{Fe}, 21.65 .+\mathrm{f}$

lution due to the tensor force in this Letter. It is well known that the one-pion exchange process is the major origin of the tensor force, which is written as

$$
V_{T}=\left(\vec{\tau}_{1} \cdot \vec{\tau}_{2}\right)\left(\left[\vec{s}_{1} \vec{s}_{2}\right]^{(2)} \cdot Y^{(2)}\right) f(r),
$$

where $\vec{\tau}_{1,2}\left(\vec{s}_{1,2}\right)$ denotes the isospin (spin) of nucleons 1 and $2,[]^{(K)}$ means the coupling of two operators in the brackets to an angular momentum (or rank) $K, Y$ denotes the spherical harmonics for the Euler angles of the relative coordinate, and the symbol $(\cdot)$ means a scalar product. Here, $f(r)$ is a function of the relative distance, $r$. Equation (1) is equivalent to the usual expression containing the $S_{12}$ function. Because the spins $\overrightarrow{s_{1}}$ and $\overrightarrow{s_{2}}$ are dipole operators and are coupled to rank 2, the total spin $S$ of two interacting nucleons must be $S=1$. If both of the bra and ket states of $V_{T}$ have $L=0$, with $L$ being the relative orbital angular momentum, their matrix element vanishes because of the $Y^{(2)}$ coupling. These properties are used later.

The (spherical) bare single-particle energy of an orbit $j$ is given by its kinetic energy and the effects from the inert core (closed shell) on the orbit $j$. As some nucleons are added to another orbit $j^{\prime}$, the single-particle energy of the orbit $j$ is changed. The nucleons on $j^{\prime}$ can form various many-body states, but we are interested in monopole effects independent of details of such many-body states. The monopole component of an interaction, $V$, is [8]:

$$
V_{j, j^{\prime}}^{T}=\frac{\sum_{J}(2 J+1)\left\langle j j^{\prime}|V| j j^{\prime}\right\rangle_{J T}}{\sum_{J}(2 J+1)},
$$

where $\left\langle j j^{\prime}|V| j j^{\prime}\right\rangle_{J T}$ stands for the (diagonal) matrix element of a state where two nucleons are coupled to an angular momentum $J$ and an isospin $T$. In the summation in Eq. (2), $J$ takes values satisfying antisymmetrization. We then construct a two-body interaction, called $V_{M}$, consisting of two-body matrix elements $V_{j, j^{\prime}}^{T}$ in Eq. (2). 
Because the $J$ dependence is averaged out in Eq. (2), the monopole interaction, $V_{M}$, represents the angular-free, i.e., monopole property of the original interaction, $V$, while it still depends on the isospin. If neutrons occupy $j^{\prime}$ and one looks into the orbit $j\left(\neq j^{\prime}\right)$ as a proton orbit, the shift of the single-particle energy of $j$ is given by

$$
\Delta \epsilon_{p}(j)=\frac{1}{2}\left\{V_{j, j^{\prime}}^{T=0}+V_{j, j^{\prime}}^{T=1}\right\} n_{n}\left(j^{\prime}\right),
$$

where $n_{n}\left(j^{\prime}\right)$ is (the expectation value of) the number of neutrons in the orbit $j^{\prime}$. The same is true for $\Delta \epsilon_{n}(j)$ as a function of $n_{p}\left(j^{\prime}\right)$. The monopole effects from orbits $j^{\prime}, j^{\prime \prime}, \ldots$ are added as these orbits are filled. The singleparticle energy, including this monopole effect, is called the effective single-particle energy (ESPE), and it depends on the configurations. We shall discuss, in this Letter, how the ESPE of an orbit $j$ varies due to the tensor force as an orbit $j^{\prime}$ is filled.

If the orbit $j^{\prime}$ is fully occupied by neutrons in Eq. (3), only the monopole effect remains over the other multipoles and Eq. (3) gives the shift of the bare single-particle energy for this shell closure. If protons and neutrons are occupying the same orbit, the change of ESPE becomes slightly more complicated due to isospin symmetry [8].

We begin with cases like Fig. 1: with orbital angular momenta being denoted by $l$ or $l^{\prime}$, protons are in either $j_{>}=l+1 / 2$ or $j_{<}=l-1 / 2$, while neutrons are in either $j_{>}^{\prime}=l^{\prime}+1 / 2$ or $j_{<}^{\prime}=l^{\prime}-1 / 2$. In examples to be discussed, these orbits represent valence or hole states near the Fermi surface, and their radial wave functions are given by the harmonic oscillator potential for simplicity.

From now on, $V$ is the tensor force. For the orbits $j$ and $j^{\prime}$, the following identity can be derived,

$$
\left(2 j_{>}+1\right) V_{j_{>}, j^{\prime}}^{T}+\left(2 j_{<}+1\right) V_{j_{<, j^{\prime}}}^{T}=0,
$$

where $T=0$ and 1 , and $j^{\prime}$ is either $j_{>}^{\prime}$ or $j_{<}^{\prime}$. Note that this identity is in the isospin formalism, and can be applied not only to cases like Fig. 1(a) but also to cases between neutrons or between protons. The identity in Eq. (4) can be proved by angular momentum algebra by summing all spin and orbital magnetic substates for the given $l$. It is

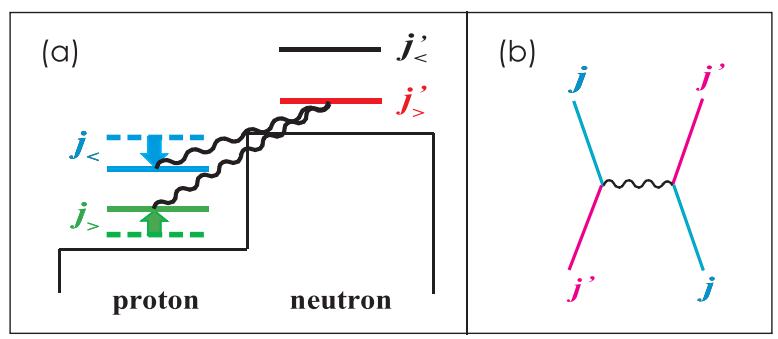

FIG. 1 (color). (a) Schematic picture of the monopole interaction produced by the tensor force between a proton in $j_{>,<}=$ $l \pm 1 / 2$ and a neutron in $j_{>,<}^{\prime}=l^{\prime} \pm 1 / 2$. (b) Exchange processes contributing to the monopole interaction of the tensor force. assumed that the radial wave function is the same for $j_{>}$ and $j_{<}$orbits, which is exactly fulfilled in the harmonic oscillator and practically so in other models if the orbits are well bound. This identity does not hold if the singleparticle state $j_{>}$or $j_{<}$is identical to $j^{\prime}$ (as excluded in Fig. 1), because the substate summation is affected by the isospin symmetry. However, the actual monopole matrix elements follow the relation in Eq. (4) semiquantitatively. One can prove that $V_{j, j^{\prime}}^{T}=0$ for $j$ or $j^{\prime}=s_{1 / 2}$. Equation (4) suggests that if both $j_{>}$and $j_{<}$orbits are fully occupied, their total tensor monopole effect vanishes.

Only exchange processes in Fig. 1(b) contribute to $V_{M}$ for the tensor force, while its direct contribution vanishes. The same property holds for a spin-spin central interaction [9]. If only exchange terms remain, the spin-coordinate part of the $T=0$ and 1 matrix elements are just opposite. Combining this with $\left(\vec{\tau}_{1} \cdot \vec{\tau}_{2}\right)$ in Eq. (1), one obtains

$$
V_{j, j^{\prime}}^{T=0}=3 \times V_{j, j^{\prime}}^{T=1} \quad \text { for } j \neq j^{\prime}
$$

Thus, the proton-neutron tensor monopole interaction is twice as strong as the $T=1$ interaction.

The question is the way in which the tensor force drives ESPE's, and whether there is a general rule for this movement. The answer is given in an intuitive way. In Fig. 2(a), a nucleon on $j_{<}$is colliding with another on $j_{>}^{\prime}$. Because of the high relative momentum between them, the spatial wave function of their relative motion is narrowly distributed in the direction of the collision which is basically the direction of the orbital motion. The spins of two nucleons are parallel in this case, giving rise to basically $S=1$. The ellipse in Fig. 2(a) represents such relative-motion wave function being spread more along the total $\operatorname{spin} S=1$. This is analogous to the case of the deuteron, and the tensor force works attractively. The same mechanism holds for two nucleons in $j_{>}$and $j_{<}^{\prime}$. On the other hand, as in Fig. 2(b), the tensor force produces a repulsive effect for two nucleons in $j_{>}$and $j_{>}^{\prime}$ (or vice versa), because the wave function of the relative motion is stretched in the direction of the collision. Thus, we can obtain a robust picture that $j_{<}$and $j_{>}^{\prime}$ (or vice versa) orbits attract each other, whereas $j_{>}$and $j_{>}^{\prime}\left(\right.$ or $j_{<}$and $j_{<}^{\prime}$ ) repel each other. In this picture, it is supposed that the tensor force being

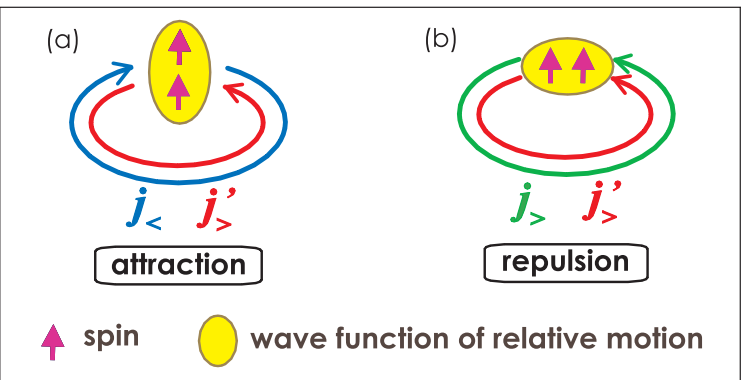

FIG. 2 (color). Intuitive picture of the tensor force acting two nucleons on orbits $j$ and $j^{\prime}$. 
discussed does not differ much from the one for the deuteron.

The radial wave functions of the two orbits must be similar in order to have a large overlap in the radial direction. A narrow spacial distribution is favored in the radial direction, in order to have a "deuteronlike" shape. This is fulfilled if the two orbits are both near the Fermi energy, because their radial wave functions have a rather sharp peak at the surface. If the radial distributions of the two orbits differ, not only does the overlap become smaller but also the relative spacial wave function is stretched in the radial direction, which is against the deuteronlike shape, making the effect less pronounced. Note that for the same radial condition, larger $l$ and $l^{\prime}$ enhance the tensor monopole effect in general, as their relative momentum becomes higher (see Fig. 2).

We assess the effect quantitatively by using a reasonable tensor force. Figure 3 exhibits the triplet-even potential due to the tensor force in potential models such as $\pi$ exchange, $\pi+\rho$ exchange, M3Y [10], AV8 ${ }^{\prime}$ [4], and the $G$ matrix (GM) for normal nuclear density. The first two are fixed from standard meson-nucleon coupling constants [11,12]. Although there are large differences in the short distance part, these potentials do not differ much for $r>0.8 \mathrm{fm}$ except for $\pi$ exchange. Since two nucleons interacting through the tensor force are not in a relative $L=0$ state (as discussed earlier), the differences at short distance are irrelevant in the following discussions. We use the $\pi+\rho$ exchange potential with a radial (inner) cutoff at $0.7 \mathrm{fm}$, for simplicity. In fact, all these interactions but $\pi$ exchange produce quite similar results. Since the $\mathrm{AV} 8^{\prime}$ interaction can reproduce the deuteron properties [4], the present tensor force should be consistent with the structure of the deuteron.

Figures 4(a)-4(d) show ESPE's as a function of $Z$ or $N$. Note that the ESPE is plotted relative to a certain orbit in cases (a) and (c). As more protons or neutrons are added, the ESPE is changed by the tensor force and by other

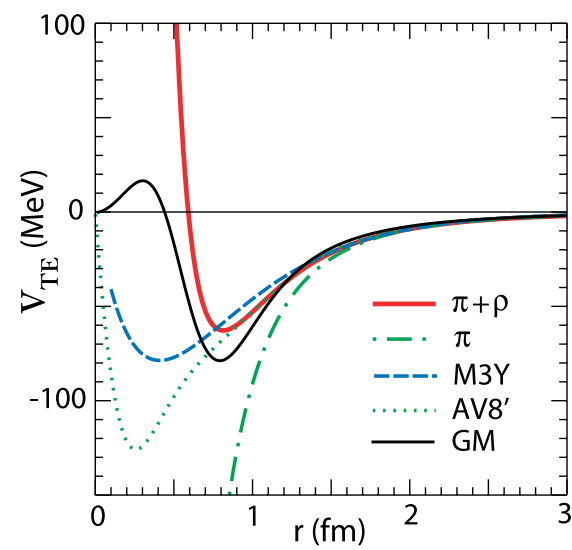

FIG. 3 (color). Triplet-even potential due to the tensor force for various interaction models. forces. The latter effect can be estimated by a WoodsSaxon potential, and appears to be rather common among the orbits in each group (a)-(d) within the given range of $Z$ or $N$. By looking at relative ESPE's [as in (a) and (c)], one can remove the common change and thus can see the tensor effect more directly.

Figure 4(a) shows ESPE's of the proton $1 d_{5 / 2}$ and $2 s_{1 / 2}$ states relative to $1 d_{3 / 2}$ as a function of $N$. As more neutrons occupy the $1 f_{7 / 2}$ orbit, these proton orbits are shifted. In Fig. 4(a), the changes due to the tensor force are indicated starting from experimental energies for ${ }^{40} \mathrm{Ca}$. Following the rule discussed with Fig. 2, the monopole interaction between proton $d_{3 / 2}$ and neutron $f_{7 / 2}$ is attractive, whereas that between proton $d_{5 / 2}$ and neutron $f_{7 / 2}$ is repulsive. Hence, as more neutrons occupy $1 f_{7 / 2}$, the proton $1 d_{3 / 2}$ goes down while $1 d_{5 / 2}$ comes up. Since the energies are shown relative to $1 d_{3 / 2}$, as $N$ increases, $2 s_{1 / 2}$ approaches $1 d_{3 / 2}$ and the splitting between $1 d_{5 / 2}$ and $1 d_{3 / 2}$ becomes narrower. A compilation of experimental data is included in Fig. 4(a) [13], showing the decreasing spacing between $1 d_{3 / 2}$ and $2 s_{1 / 2}$ in agreement with the calculation. The situation is more open for $1 d_{5 / 2}$, because of greater ambiguity due to deep hole states.
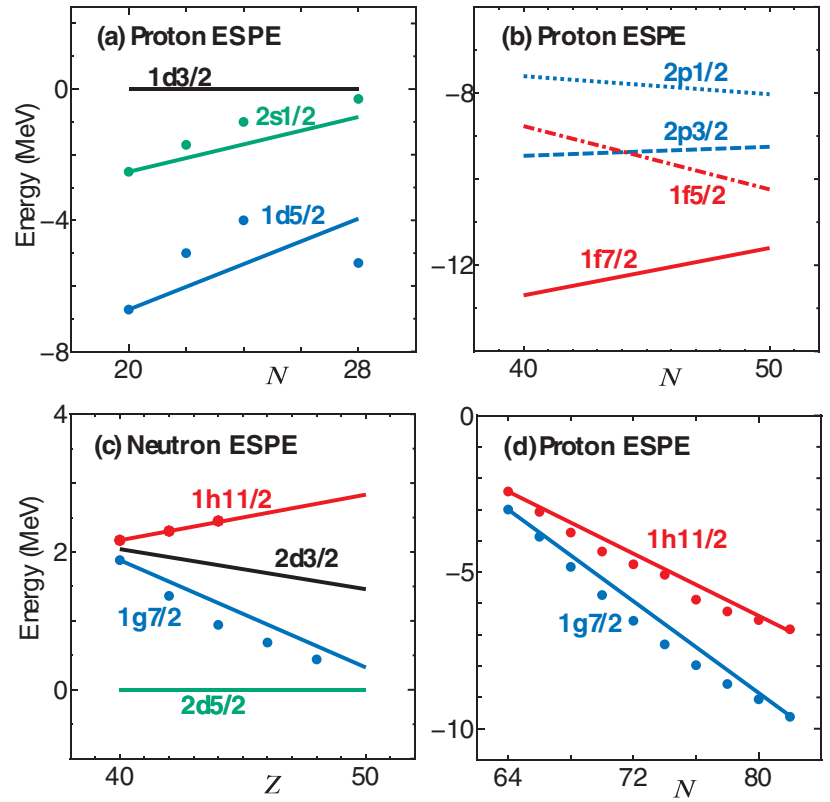

FIG. 4 (color). Proton (neutron) ESPE as a function of $N(Z)$. Lines in (a)-(c) show the change of ESPE's calculated from the $\pi+\rho$ tensor force. Points represent the corresponding experimental data. (a) Proton ESPE's in Ca isotopes relative to $1 \mathrm{~d}_{3 / 2}$. Points are from [13]. (b) Proton ESPE's in Ni isotopes; calculations only. See [19] for related experimental data. (c) Neutron ESPE's in $N=51$ isotones relative to $2 d_{5 / 2}$; points are from [21]. (d) Proton ESPE's in Sb isotopes; points are from [18]. Lines include a common shift of ESPE as well as the tensor effect (see the text). 
Figure 4(b) exhibits proton $p f$-shell orbits from ${ }^{68} \mathrm{Ni}$ to ${ }^{78} \mathrm{Ni}$ as neutrons occupy the $1 g_{9 / 2}$ orbit. The ESPE's for ${ }^{68} \mathrm{Ni}$ predicted by the shell model with the GXPF1 interaction [14] are used as the starting point, and the changes due to the tensor force are shown. Again as in Fig. 1(a), proton $1 f_{5 / 2}$ is pulled down while $1 f_{7 / 2}$ is lifted up, as $N$ increases. The $Z=28$ gap becomes rather small at ${ }^{78} \mathrm{Ni}$, and the sequence of the orbits is quite different between ${ }^{68} \mathrm{Ni}$ and ${ }^{78} \mathrm{Ni}$ [15]. Other monopole effects are likely to be rather common among the $p f$-shell orbits as discussed above. Thus, the predictions shown in Fig. 1(b) may produce exciting features of exotic Ni isotopes. In fact, unusually low-lying $2^{+}$states are known [16].

From $Z=40$ to 50 , the $1 g_{9 / 2}$ orbit is filled by protons. Through the tensor force, these protons lower the neutron $1 g_{7 / 2}$, while they push up the $1 h_{11 / 2}$. In Fig. 4(c), such changes of ESPE's due to the tensor force are shown starting from experimental values at $Z=40$. The growing spacing between neutron $1 g_{7 / 2}$ and $1 h_{11 / 2}$ is explained well. This lowering of $1 g_{7 / 2}$ is the phenomenon pointed out by Federman and Pittel [17]. The lowering was attributed to a large overlap between proton $1 g_{9 / 2}$ and neutron $1 g_{7 / 2}$, but the same mechanism should lower neutron $1 h_{11 / 2}$ which has a large radial overlap of 0.98 with $1 g_{9 / 2}$ for the harmonic oscillator, contrary to experiment.

Figure 4(d) shows proton $1 h_{11 / 2}$ and $1 g_{7 / 2}$ ESPE's as a function of $N$ [18]. In Fig. 4(d), their changes due to the tensor force are indicated starting from experimental values for $N=64$. Here, in order to shed a light on the increasing spacing between $1 h_{11 / 2}$ and $1 g_{7 / 2}$ in the full energy scale of ESPE's, a common monopole shift for both $1 h_{11 / 2}$ and $1 g_{7 / 2},-0.3 \Delta N(\mathrm{MeV})$ is added to the tensor effect so as to accommodate other monopole effects. This common shift is more or less what is obtained from WoodsSaxon potentials with reasonable parameters. As more neutrons occupy $1 h_{11 / 2}$, the proton $1 h_{11 / 2}$ and $1 g_{7 / 2}$ move apart due to the tensor force. The agreement with experiment on this feature is remarkable. We note that this case does not belong to the one in Eq. (3). In the present case, all magnetic substates of the neutron $1 h_{11 / 2}$ orbit are occupied, to a good approximation, with equal probability due to the strong pairing correlation. It can then be proved that Eq. (3) is still valid. The neutron occupation of $1 h_{11 / 2}$ is assumed to increase uniformly from $N=64$ to 82 , and the contributions from the other orbits are assumed to be minor.

Since the tensor force is fixed from the underlying $N N$ interaction, the calculations in Fig. 4 are all parameter free. There are many other observed cases $[15,19]$ to which the tensor shell evolution is relevant, for instance, the neutron $1 d_{5 / 2}-2 s_{1 / 2}$ inversion between ${ }^{15} \mathrm{C}$ and ${ }^{17} \mathrm{O}$.

In summary, the shell evolution due to the tensor force was presented with the underlying mechanism. A relevant identity was shown, and an intuitive explanation was given.
The tensor force produces general and robust effects on the shell and (sub-)magic structures from the $p$ shell to the superheavy regions. The significant role of the tensor force as a direct consequence of $\pi$ and $\rho$ meson exchange can be related to the chiral perturbation idea of Weinberg [20]. Indeed, the long-range part of the $N N$ interaction manifests itself quantitatively in the single-particle spectra in a unique and systematic way. Since the tensor force plays such significant roles, its multipole components should be further investigated.

The strongly attractive proton-neutron $j_{>}-j_{<}$coupling has been pointed out as the origin of the shell evolution within one major shell in [9]. The tensor effect includes this case [see Fig. 4(c)], but it can be due to the $\sigma \sigma \tau \tau$ central force, too [9]. Although the relation between these two forces is an intriguing subject to be clarified, Fig. 4(c) may be a hint that the tensor force is the major origin.

This work was supported in part by a Grant-in-Aid for Specially Promoted Research (No. 13002001) from the MEXT. This work has been a part of the RIKEN-CNS joint research project on large-scale nuclear-structure calculations. The authors acknowledge Drs. M. Serra, W. B. Walters, and A. Gelberg for valuable help.

*Electronic address: otsuka@phys.s.u-tokyo.ac.jp

[1] M. G. Mayer, Phys. Rev. 75, 1969 (1949); O. Haxel, J.H. D. Jensen, and H.E. Suess, Phys. Rev. 75, 1766 (1949).

[2] H. Yukawa, Proc. Phys. Math. Soc. Japan 17, 48 (1935).

[3] A. Arima and T. Terasawa, Prog. Theor. Phys. 23, 115 (1960).

[4] B. S. Pudliner et al., Phys. Rev. C 56, 1720 (1997).

[5] D. J. Millener and D. Kurath, Nucl. Phys. A255, 315 (1975).

[6] R. R. Scheerbaum, Phys. Lett. 63B, 381 (1976).

[7] A. L. Goodman and J. Borysowicz, Nucl. Phys. A295, 333 (1978).

[8] R. K. Bansal and J. B. French, Phys. Lett. 11, 145 (1964).

[9] T. Otsuka et al., Phys. Rev. Lett. 87, 082502 (2001); T. Otsuka, Prog. Theor. Phys. Suppl. 146, 6 (2002).

[10] G. Bertsch, J. Borysowicz, H. McManus, and W. G. Love, Nucl. Phys. A284, 399 (1977).

[11] F. Osterfeld, Rev. Mod. Phys. 64, 491 (1992).

[12] S.-O. Bäckman, G.E. Brown, and J.A. Niskanen, Phys. Rep. 124, 1 (1985).

[13] P. D. Cottle and K. W. Kemper, Phys. Rev. C 58, 3761 (1998).

[14] M. Honma, T. Otsuka, B. A. Brown, and T. Mizusaki, Phys. Rev. C 69, 034335 (2004).

[15] H. Grawe et al., Eur. Phys. J. A 25, 357 (2005).

[16] M. Sawicka et al., Phys. Rev. C 68, 044304 (2003); Eur. Phys. J. A 20, 109 (2003).

[17] P. Federman and S. Pittel, Phys. Lett. 69B, 385 (1977).

[18] J. P. Schiffer et al., Phys. Rev. Lett. 92, 162501 (2004).

[19] H. Grawe, Springer Lect. Notes in Phys. 651, 33 (2004).

[20] S. Weinberg, Phys. Lett. B 251, 288 (1990).

[21] H. Grawe et al., Nucl. Phys. A704, 211c (2002). 